\title{
Radiation hardness studies and detector characterisation at the JSI TRIGA reactor
}

\author{
L.Snoj ${ }^{1,3}$ (luka.snoj@ijs.si), K. Ambrožič ${ }^{1}$, A. Čufar ${ }^{1}$, T. Goričanec ${ }^{1}$, A. Jazbec ${ }^{1}$, I. Lengar ${ }^{1}$, A. Pungerčič ${ }^{1}$, \\ V. Radulović ${ }^{1}$, S. Rupnik ${ }^{1}$, Ž. Štancar ${ }^{1}$, G. Žerovnik ${ }^{1}$, A. Žohar ${ }^{1}$, V. Cindro ${ }^{1}$, G. Kramberger ${ }^{1}$, I. Mandić1, \\ M. Mikuž ${ }^{1,3}$, L. Barbot ${ }^{2}$, H. Carcreff ${ }^{2}$, C. Destouches ${ }^{2}$, D. Fourmentel ${ }^{2}$, A. Gruel ${ }^{2}$ and J.F. Villard ${ }^{2}$ \\ ${ }^{1}$ Jozef Stefan Institute, Jamova cesta 39, 1000 Ljubljana, Slovenija \\ ${ }^{2}$ CEA, DEN, Cadarache, DER/SPESI, F-13108 Saint Paul lez Durance, France \\ ${ }^{3}$ Faculty of mathematics and physics, Uniuversity of Ljubljana, Jadranska cesta 19 Ljubljana, Slovenija
}

\begin{abstract}
The JSI TRIGA reactor features several in-core and ex-core irradiation facilities, each having different properties, such as neutron/gamma flux intensity, spectra and irradiation volume. A series of experiments and calculations was performed in order to characterise radiation fields in irradiation channel thus allowing users to perform irradiations in a well characterised environment. Since 2001 the reactor has been heavily used for radiation hardness studies for components used at accelerators such as the Large Hadron Collider (LHC) at CERN. Since 2010 it has been extensively used for testing of new detectors and innovative data acquisition systems and methods developed and used by the CEA. Recently, several campaigns were initiated to characterise the gamma field in the reactor and use the experimental data for improvement of the treatment of delayed gammas in Monte Carlo particle transport codes. In the future it is planned to extend the testing options by employing pulse mode operation, installation of a high energy gamma ray irradiation facility and allow irradiation of larger samples at elevated temperature.
\end{abstract}

Key words: TRIGA, radiation hardness, detectors, testing, Nuclear measurements, Neutron radiation effects, Gamma-ray effects, Reactor instrumentation

\section{INTRODUCTION}

$\mathrm{T}_{0}$ he Jožef Stefan Institute (JSI) TRIGA reactor has been operating since 1966. It features two modes of operation; i.e. steady state at $250 \mathrm{~kW}$ and pulse mode at up to $1 \mathrm{GW}$. Until the 1990s it was mainly used for neutron activation analysis (NAA), isotope production, and training. Since 1990, isotope production declined and was replaced by irradiations for radiation hardness studies for components used at accelerators such as the Large Hadron Collider (LHC) at CERN. The first characterisation of the irradiation fields in the reactor was performed experimentally. With the appearance of faster and cheaper computers at the end of the 1990s the experimental characterisation was supported by calculations. More than a decade of efforts in experimental and computational characterisation of the neutron and gamma fields in the irradiation facilities resulted in JSI TRIGA reactor being a very well characterised neutron irradiation facility. For this reason it has become the reference centre for neutron radiation hardness studies for the CERN's LHC and other accelerators within the H2020 project AIDA II (Advanced European Infrastructures for Detectors at Accelerators). Several companies (ITER, National instruments, ISEC, Dito lighting, Nanocut, etc.) have been using the reactor for testing of radiation hardness of their products, such as data acquisition and processing systems, surveillance cameras, LED lighting, and similar. In addition, the reactor has been used for testing various detectors such as fission chambers and self-powered neutron detectors (SPNDs) as well as innovative data acquisition systems and methods developed and used by the CEA.

Since 2010 the JSI TRIGA reactor radiation field characterization shifted from neutron to gamma radiation. This led to several JSI-CEA experimental campaigns on gamma field measurements inside the reactor during several stages of operation (steady state operation and after shutdown) by various detector types such as miniature ionization chambers (MIC), TLDs (Thermo-Luminescent Dosimeters) and OSLDs (Optically Stimulated Luminescent Dosimeters) and gamma calorimeters using ITER relevant sample materials such as Eurofer, W, Nb3Sn, etc. In the near future we plan to install a water activation loop enabling gamma irradiation with $6 \mathrm{MeV}$ and $7 \mathrm{MeV}$ gamma rays from ${ }^{16} \mathrm{~N}$. In the paper we describe the abovementioned activities.

\section{IRRADIATIONS AT THE JSI TRIGA REACTOR}

\section{A. Reactor description}

The JSI TRIGA Mark II reactor is a typical TRIGA type reactor. It is a pool type reactor, cooled by natural convection, capable of operating maximal steady thermal power of $250 \mathrm{~kW}$ and up to $1 \mathrm{GW}$ in pulse mode. The reactor core has an annular 
configuration with a diameter of approx. $44 \mathrm{~cm}$, with 91 in-core positions for fuel elements, 4 control rods, and numerous irradiation positions (Fig. 1). Nuclear fuel is a homogeneous mixture of fuel and moderator in form of uranium zirconium hydride (U-ZrH).

The reactor usually operates with the central position (A1 or $\mathrm{CC}$ ), and most of the F-ring positions empty and available for irradiations and two control rods (Transient and Safety) completely withdrawn. In addition to standard reactor in-core positions, tube with a triangular cross-section serves as an irradiation position called Triangular channel (TriC) (Figure 1).

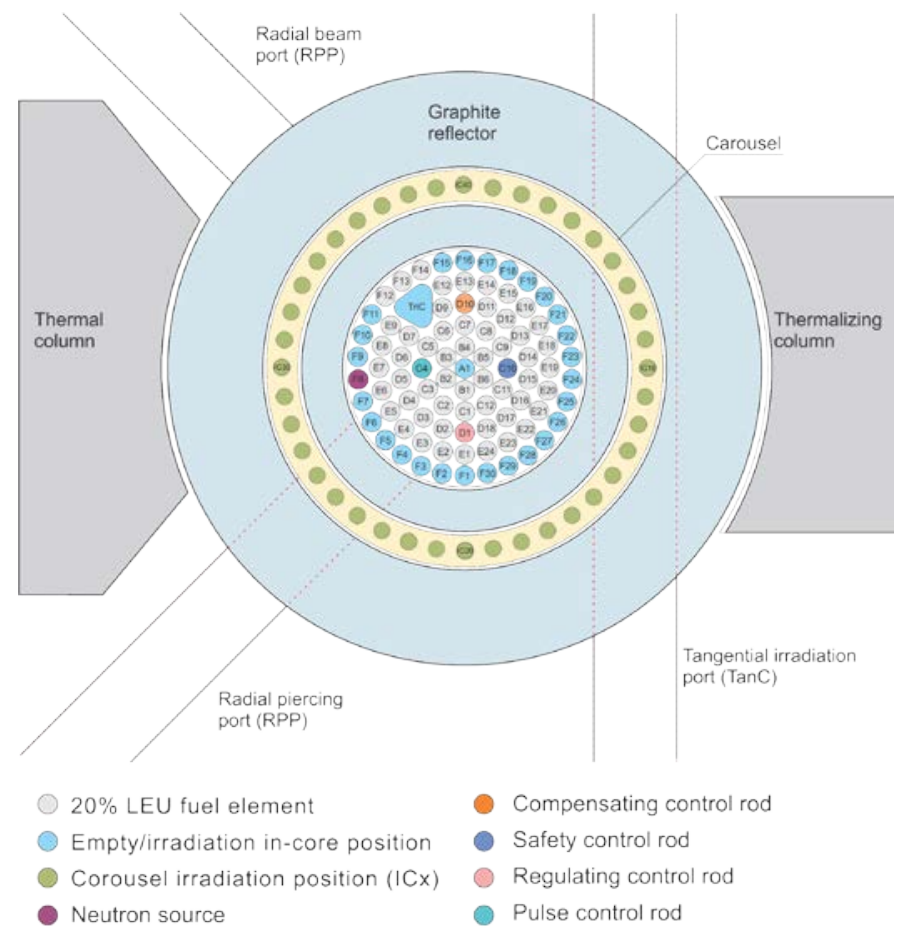

Figure 1. JSI TRIGA reactor core schematics with smaller irradiation facilities pointed out.

The JSI TRIGA is also equipped with numerous ex-core irradiation positions. The first set of ex-core irradiation positions is located in the carousel, a rotary device inside the graphite reflector, holding 40 cylindrical irradiation positions (ICx) with $3 \mathrm{~cm}$ in diameter (Fig. 1). There are also several larger horizontal channels that can be utilised for irradiation of larger samples. Two cylindrically shaped irradiation channels with an internal diameter of $15.4 \mathrm{~cm}$ extend radially outwards from the core (Fig. 2). The Radial Piercing Port (RPP) pierces the reactor graphite reflector, while the Radial Beam Port (RBP) does not. Additionally, two channels with the same diameter, are available. The first, named Tangential channel (TanC), also pierces the graphite reflector and is shifted 32.4 $\mathrm{cm}$ to the side and $9.4 \mathrm{~cm}$ below the reactor core centre. The second, named Thermal irradiation port (TIP), pierces the Thermal column and is shifted $106 \mathrm{~cm}$ to the other side of the core and elevated by $21.6 \mathrm{~cm}$ compared to the Tangential channel (Fig. 2).

The two largest irradiation positions extend outside of the biological shield, and are predominantly used for neutron irradiation (Figs. 2, 3). The Dry chamber is a small room inside the reactor biological shield, shielded with heavy concrete doors, into which neutrons are guided through a graphite stack and lead shielding, the Thermalizing column. The Thermal column is composed of a larger graphite stack, shielded by heavy concrete doors. Neutrons can be conveyed into a labyrinth structure constructed around the Thermal column, composed of borated paraffin and barite concrete blocks, to prevent excess radiation levels inside the reactor hall.

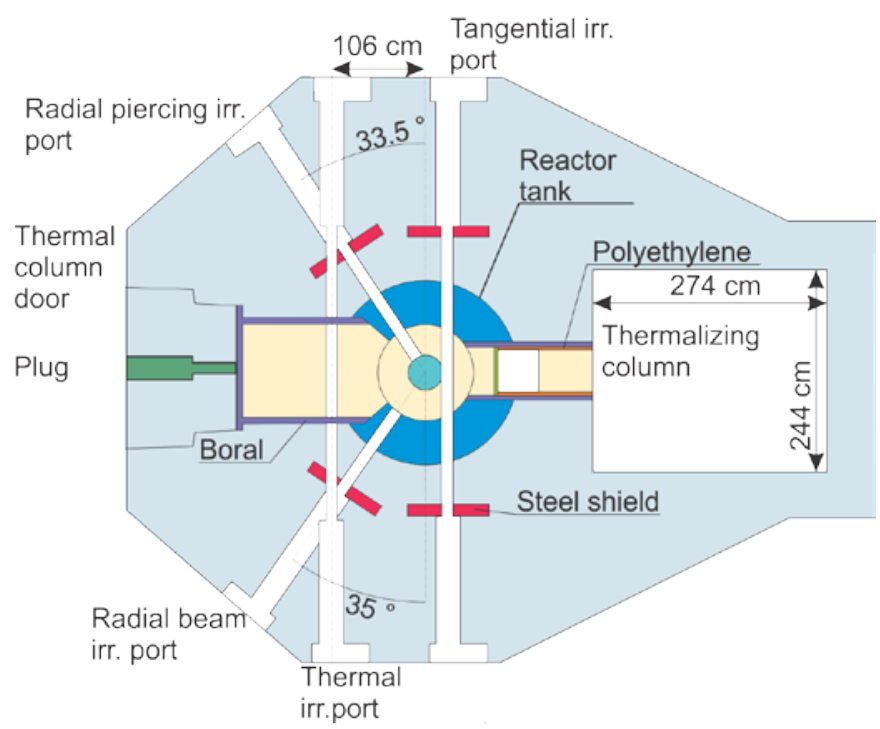

Figure 2. JSI TRIGA reactor structure with horizontal irradiation ports and a thermalizing column.

\section{B. Characterisation of irradiation fields}

The first computational model of the JSI TRIGA reactor was made in the TRIGLAV [1] code for the purpose of burn-up calculations, required for core management. It was a $2 \mathrm{D}$ model allowing neutron flux calculations in 4 energy groups. The second computational model was made in the Monte Carlo particle transport code MCNP [2] (ref.) for the purpose of criticality calculations of criticality benchmark experiments performed in 1991 [3-4]. In 2006, a project was initiated to develop a methodology to quantitatively determine the spectral characteristics of the irradiation facilities by a combination of computational and experimental techniques. Computational methods, in particular full 3D models of the facility using Monte Carlo methods were used, which are very powerful in providing information on the neutron flux energy spectrum. The calculated results require careful validation to eliminate modelling errors, biases due to model simplifications, etc. In order to verify and validate the computational methods for neutron flux calculation in the TRIGA research reactor, a series of experiments has been performed.

The neutron activation method was used to experimentally validate the calculated reaction rate distributions in the TRIGA reactor. In the experiment, aluminium-gold (99.9 wt. \% Al, 0.1 wt. $\% \mathrm{Au}$ ) foils were irradiated in 33 locations; 6 in the core and 27 in the Carousel facility in the reflector [5]. The experimental results were compared to the calculations performed with Monte Carlo code MCNP using a detailed geometrical model of the reactor. In 2008 a collaboration between the JSI and the CEA was initiated. The purpose of the collaboration was to 
further characterize the reactor neutron radiation field by means of miniature fission chambers $\left({ }^{235} U\right.$ and $\left.{ }^{238} U\right)$ [6] and activation of different neutron dosimeters (Al-Au, Al-Mn, Al-U, Al-Co, Al-Sc, Al-Th, Sn enriched in ${ }^{117} \mathrm{Sn}, \mathrm{Ni}$ and Fe) [7-10].

The fission chambers were used to measure the axial fission rate profiles in various radial positions in the core. This was performed for different control rod positions, which significantly tilt the neutron flux profile [11-13]. Another feature of these measurements was that they provided absolute values of reaction rates at certain positions, meaning that they could be used not only for the validation of calculated reaction rate profiles but also for the validation of the normalisation procedure with respect to the neutron source intensity or the reactor power level [14]. These measurements were later used as experimental benchmark experiments and were published in the 2017 edition of the International Handbook of Evaluated Reactor Physics Experiments

Axial reaction rate profile measurements were performed also with $\mathrm{Au}$ foils [15]. The agreement between measured and calculated $(\mathrm{n}, \gamma)$ reaction rate profile on $198 \mathrm{Au}$ was very good thus confirming validity of the computational model.

Since 2010 the reactor has been used also as a strong source of gamma rays for gamma radiation hardness studies. This led to several JSI-CEA joint experimental campaigns to measure the gamma field inside the reactor, both during operation and in shut down conditions. The first measurements were performed using a CEA-developed miniature ionisation chamber (MIC). At full reactor power a discrepancy of around $50 \%$ was observed between the measured and the calculated detector currents; in the latter taking into consideration only the prompt gamma field. Further experimental measurements of MIC currents following reactor SCRAMs (reactor shut down with rapid insertions of control rods) provided evidence that over 30 $\%$ of the total measured signal is due to the delayed gamma field, originating from fission and activation products, which are untreated in the calculations [16-17].

In 2016 a CEA-JSI project was initiated, within which the delayed and prompt gamma field in the reactor is being measured by TLDs (Thermo-Luminescent Dosimeters) and OSLDs (Optically Stimulated Luminescence Dosimeters) as well as ionisation chambers [18]. It was found that the delayed gamma contribution ranges from $31.4 \pm 2.8 \%$ in the reactor core center, decreasing linearly to $18.9 \pm 2.0 \%$ at the outermost core ring [19]. All these data will serve as reference data for the improvement of the treatment of delayed gammas in the Monte Carlo particle transport codes [20].

The experimentally validated computational models were used to calculate neutron and gamma fluxes, spectra and dose rates in all JSI TRIGA irradiation facilities. The results are presented in Tables 1, 2 and Figures 3, 4.

Table 1. Calculated neutron and prompt gamma fluxes in selected JSI TRIGA irradiation facilities at $250 \mathrm{~kW}$

\begin{tabular}{|l|l|l|}
\hline & Neutron flux $\left[\mathrm{cm}^{-2} \mathrm{~s}^{-1}\right]$ & $\begin{array}{l}\text { Gamma flux }\left[\mathrm{cm}^{-}\right. \\
\left.{ }^{2} \mathrm{~s}^{-1}\right]\end{array}$ \\
\hline $\mathrm{CC}$ & $1.93 \times 10^{13}$ & $2.07 \times 10^{13}$ \\
\hline IC 40 & $2.09 \times 10^{12}$ & $1.37 \times 10^{12}$ \\
\hline TanC & $2.23 \times 10^{12}$ & $2.12 \times 10^{12}$ \\
\hline
\end{tabular}

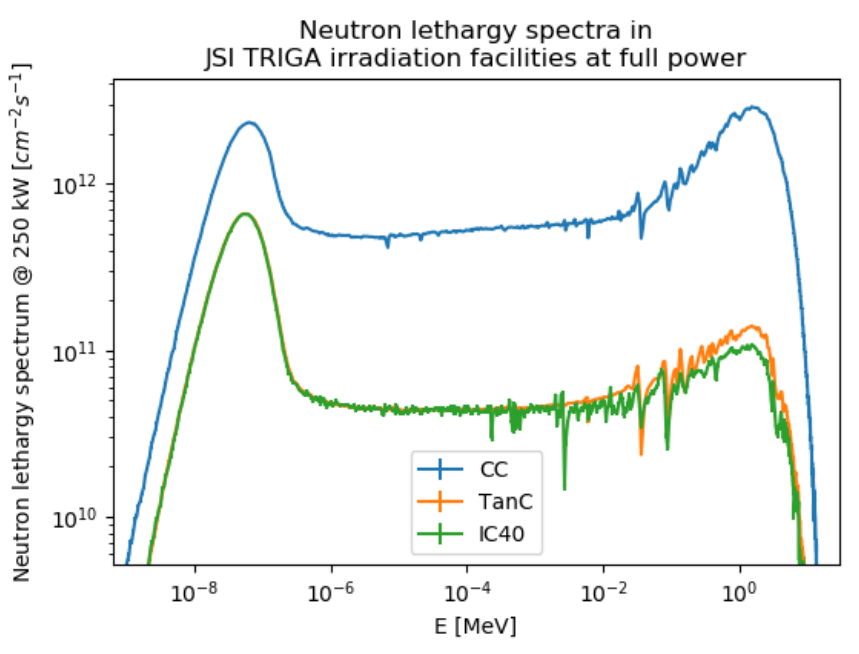

Figure 3. Neutron lethargy spectra in selected JSI TRIGA irradiation facilities at $250 \mathrm{~kW}$.

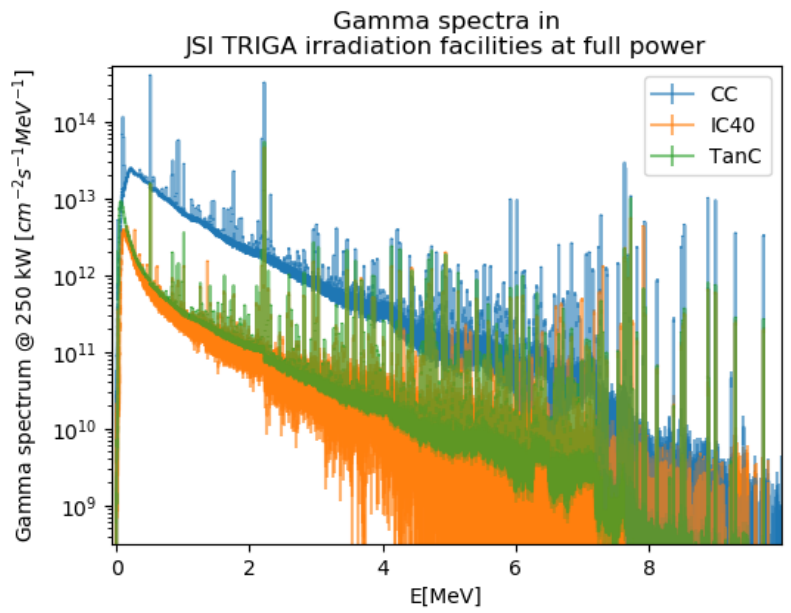

Figure 4. Gamma spectra in selected JSI TRIGA irradiation facilities at 250 $\mathrm{kW}$.

\section{Gamma irradiation}

The JSI TRIGA is lately also being utilized for irradiation in a mixed neutron and gamma-ray field during reactor operation or in a pure gamma-ray field utilizing activated nuclear fuel. Compared to typical ${ }^{137} \mathrm{Cs}$ or ${ }^{60} \mathrm{Co}$ irradiation facilities, the gamma spectrum is more representative to those in fission and fusion facilities.

Apart from the abovementioned facilities, a larger special irradiation facility (Figure 5) in form of a watertight aluminium box, with a cable guide tube for pure gamma-ray irradiation with activated nuclear has been constructed in order to accommodate gamma-ray irradiation of larger electronic assemblies which need to be connected to readout and testing equipment on the reactor platform.

The facility utilizes up to 6 activated fuel elements, which are relocated from the reactor core to the fuel element rack at the edge of the reactor tank. 
Table 2. Neutron silicon dose equivalent and gamma air kerma in selected JSI TRIGA irradiation facilities at $250 \mathrm{~kW}$.

\begin{tabular}{|l|l|l|}
\hline & $\begin{array}{c}\text { Neutron 1 MeV silicon dose } \\
\text { equivalent }\left[\mathrm{Gy} / \mathrm{h}_{\mathrm{Si}}\right]\end{array}$ & $\begin{array}{l}\text { Gamma air kerma } \\
{[\mathrm{Gy} / \mathrm{h}]}\end{array}$ \\
\hline $\mathrm{CC}$ & $8.33 \times 10^{3}$ & $2.92 \times 10^{5}$ \\
\hline IC 40 & $3.06 \times 10^{2}$ & $1.69 \times 10^{4}$ \\
\hline TanC & $4.01 \times 10^{2}$ & $2.14 \times 10^{4}$ \\
\hline
\end{tabular}

The dose rate field peak intensity and homogeneity inside the aluminium irradiation box can be controlled by adjusting the box distance from the rack, loading the rack with different fuel elements and by adjusting the reactor power level and duration for fuel element activation (Figure 6). Dose-rates of up to approximately $150 \mathrm{~Gy} / \mathrm{h}$ inside the irradiation box can be reached this way.

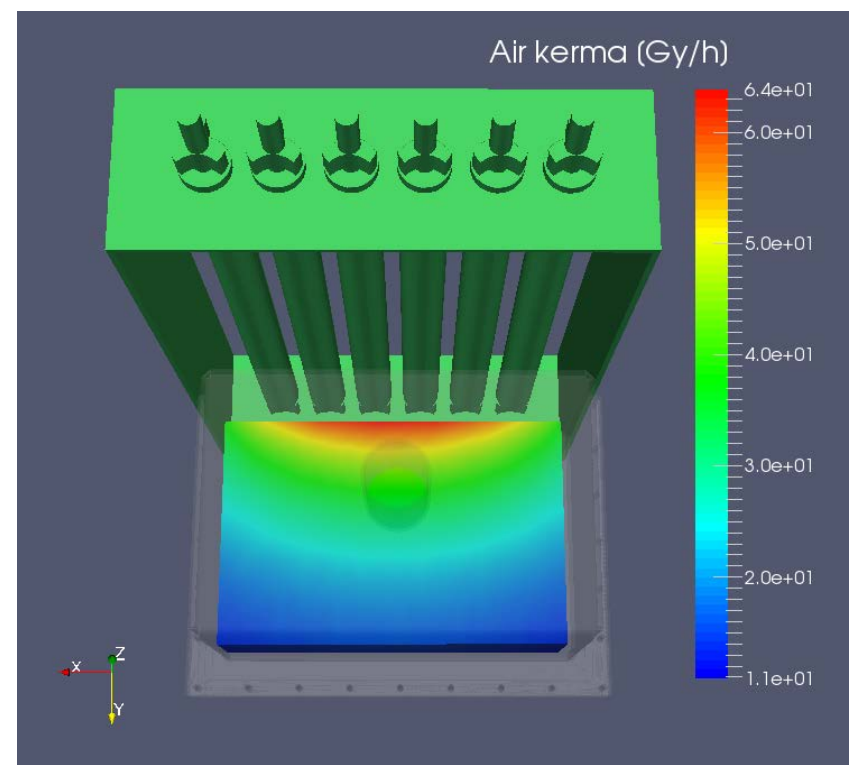

Figure 5. A visualization of air kerma rate inside of the irradiation box, using B-ring fuel elements after $1 \mathrm{~h}$ of full power reactor operation and $20 \mathrm{~min}$ cooldown.

\section{RADIATION HARDNESS STUDIES}

Since 1999 the reactor has been extensively used for irradiations of various materials with both neutrons and gamma rays. One of important activities is the testing of components for detectors for particle physics experiments built at high energy colliders - mostly Large Hadron Collider (LHC at CERN) [21] and KEK B-factory ring (KEKB at Tsukuba, Japan). Si detectors for tracking particles are the essential part of these experiments. During operation they are exposed to high fluences of energetic charged particles and neutrons. Displacement damage caused by these hadrons in silicon may deteriorate the detector performance. A program devoted to studies of radiation resistance of Si detectors was established. For irradiating the samples in the TRIGA reactor, a dedicated relatively large "triangular" channel $(6 \mathrm{~cm}$ in diameter) was constructed, which allowed testing of radiation damage of larger size Si detectors along with the associated electronics, at different temperatures by installing a heating/cooling module inside the channel. Due to well characterized irradiation channel the JSI TRIGA reactor has become an unofficial global reference centre for silicon detectors irradiation. Furthermore, the reactor has been included into the AIDA and AIDA-2020 (Advanced European Infrastructures for Detectors and Accelerators) project [22] funded by the Horizon 2020 of the European Commission. Few hundred irradiations of this kind are performed yearly for users such as CERN, DESY (German Electron Synchrotron) and KEK (High Energy Accelerator Research Organization, Japan), as well as for various universities and institutes. High energy physics experiments

Tracking detectors in HEP experiments are usually placed close to the interaction point at collider experiment and they are exposed to high fluences of outcoming particles originating from collisions. While fluences of about $1015 \mathrm{~cm}-2$ are benchmark for current experiments (ATLAS, CMS) during ten years of operation, an order of magnitude higher fluences are expected after upgrade to High Luminosity LHC in the next decade. In next generation experiments at future hadron colliders up to two orders of magnitude higher fluences are expected. While it is practically impossible to reach fluences expected in future colliders by direct exposure of devices into existing high energy proton beams (like Proton synchrotron at CERN) they can be obtained at Triga reactor within reasonable time.

Bulk damage in silicon lattice (interstitial atoms, vacancies and more complex defects) causes a change of the effective dopant concentration, an increase of leakage current and trapping of charge carriers. As a consequence, the decrease of collected charge and increase of self heating due to the leakage current are two most important effects limiting the functionality of detectors after several years of operation particle physics experiment. The dominant part of the damage at LHC experiments is caused by charged hadrons and neutrons. Their contribution to the radiation field depends on the position with respect to the interaction point. Energetic neutrons and charged hadrons both cause similar bulk damage in silicon lattice so the reactor can be used as an efficient tool for studying radiation damage.

Beside short irradiation time and relatively simple access to the irradiation channels, the exact knowledge of reactor fluxes and spectrum of neutrons is important for extensive irradiation programs. Uniformity of the neutron flux is also advantageous when compared to irradiations in particle beams, where scanning technique is frequently used due to the limited size of beam $(\leq 1 \mathrm{~cm})$.

Varieties of silicon sensors were exposed to the reactor neutrons. Silicon strip and pixel sensors with $\mathrm{p}-\mathrm{i}-\mathrm{n}$ or $\mathrm{n}-\mathrm{i}-\mathrm{p}$ structures are most commonly used for detecting particles in trackers. Studies of dependence of leakage current, effective dopant concentration and charge carrier trapping on neutron fluence are essential for successful design and operation of tracking detectors in particle physics experiments.

Good calibration and stable operation of the reactor facility enabled measurements of several very important effects of radiation on silicon detectors. For example measurements of the change of the effective dopant concentration after irradiation the violation of NIEL (non-ionizing energy loss) hypothesis was found for oxygenated sensors . In addition to already mentioned measurements of effective trapping times lower 
trapping of charge carriers was also found after irradiation with neutrons when compared to protons [23]. Another important effect for operation of detectors at high fluences and high bias voltages is the multiplication of charge carriers which was first studied in sensors irradiated at TRIGA reactor [24].

\section{A. Industry}

In the recent years the activities in the field of radiation hardness studies were expanded by collaboration with industrial partners. In collaboration with the Slovenian company Dito lighting [25] irradiation and testing of radiation hard LED lighting to be used in high radiation areas, such as particle accelerators, hot cell facilities, containment in nuclear power plants, etc. was performed. The combined efforts resulted in the world's most radiation tolerant LED light and the world's first and only LOCA (loss of coolant accident) tested and certified LED light.

In addition a new generation of cameras for radiation environments in being developed in collaboration with the Swedish company ISEC [26], supplier of monitoring systems for facilities with ionizing radiation. During the research and development process of the above items it was decided to test them in mixed (neutron + gamma) and pure gamma field separately. Pure gamma irradiation was performed by using irradiated nuclear fuel as the gamma source and custom made submersible $\mathrm{Al}$ box $(30 \mathrm{~cm} \times 20 \mathrm{~cm} \times 20 \mathrm{~cm})$ for housing the electronic components under water. In such way, the irradiation of components can be performed online that is during their operation. The dose rate in such device is approx. $100 \mathrm{~Gy} / \mathrm{h}$. During the testing the accumulated dose is measured with RADFET (radiation sensing field-effect transistor) detectors, time dependent dose rate however is measured with gas flow ionization chamber.

In the dry chamber facility we irradiated National Instruments' cRIO and PXI products and performed functional testing against thermal neutrons. This was done in collaboration with the ITER agency, the final user of the products. The aim was to determine at what dose levels there is observable degradation in analog signal performance, partial and/or temporary functional failures, permanent failure, for the entire system and individual components. It was found that failure rates were less than or equal to what was seen with fast neutron testing performed at the Frascati neutron generator.

A series of small ultrasonic transducers developed by IONIX and TWI limited, used for nuclear fuel testing have been tested for radiation resistance inside of the JSI TRIGA central channel, triangular channel and irradiation channel F19, with online monitoring of their performance.

\section{DETECTOR CHARACTERISATION}

\section{A. Nuclear instrumentation testing}

These last years, several dedicated experimental campaigns were performed in collaboration with the CEA, aimed at demonstrating and validating the functioning of neutron ad gamma detectors, for example self-powered neutron detectors
(SPNDs), miniature ionization chambers and fission chambers in representative reactor conditions [27]. These campaigns included unique tests of innovative instrumentation developed by the CEA, such as regenerative fission chambers, but also validation tests of the advanced acquisition system MONACO [28]. Additionally, some experimental results served as validation of dedicated computational schemes for the calculation of neutron sensor signal, and particularly SPND signal. The corresponding tests included several SPNDs with different material compositions and geometries; the experimental tests were performed in different locations in the core of the JSI TRIGA reactor. The entry data required in the computational schemes used to calculate the detector response signals, i.e. the neutron and gamma flux levels and energy spectra in close vicinity of the tested detector assemblies, was provided by the JSI. The calculations were performed by explicitly modelling the tested detector assemblies and including them in a detailed, verified and validated computational model of the JSI TRIGA reactor. The neutron flux levels were verified and / or adjusted on the basis of measurements for the ${ }^{197} \mathrm{Au}(\mathrm{n}, \gamma)$ and ${ }^{27} \mathrm{Al}(\mathrm{n}, \alpha)$ reaction rates, sensitive to thermal and epithermal, and fast neutrons, respectively.

\section{B. SiC based detectors}

In 2016, the NATO Science for Peace and Security Programme funded research project "Engineering Silicon Carbide for Border and Port Security" - E-SiCure was launched, with the main objective of combining theoretical, experimental and applied research towards the development of radiation-hard silicon carbide $(\mathrm{SiC})$ based detectors of special nuclear materials (SNM), and by that way, to enhance border and port security barriers. Along the plan, material modification processes are employed firstly to study, and secondly to manipulate the most severe electrically active defects (which trap or annihilate free charge carriers), by specific ion implantation and defect engineering. In the framework of the ESiCure project, detectors were developed, based on $\mathrm{SiC}$ Schottky Barrier Diodes (SBDs) and ${ }^{10} \mathrm{~B}$ and ${ }^{6} \mathrm{LiF}$ as converters of thermal neutrons to charged particles, and tested in the Dry chamber of the JSI TRIGA reactor. The lateral dimensions of the $\mathrm{SiC}$ detectors were $1 \mathrm{~mm} \times 1 \mathrm{~mm}$, the active thickness varied between $25 \mu \mathrm{m}, 69 \mu \mathrm{m}$ and $170 \mu \mathrm{m}$. The detectors had a clearly measurable neutron response, which varied linearly with the incident neutron flux. The average detector sensitivity was around $10^{-5}$ counts per second (cps), per $\mathrm{n} \mathrm{cm}^{-2} \mathrm{~s}^{-1}$. Extrapolating the experimental neutron sensitivity values for single SiC SBDs to larger arrays of such detectors, e.g. $100 \times 1000$ (the dimensions being $0.2 \mathrm{~m} \times 2 \mathrm{~m}$, comparable to large $\mathrm{BF}_{3}$ or ${ }^{3} \mathrm{He}$ detectors in use for neutron monitor detectors) gives a scaled sensitivity value of the order of $1 \mathrm{cps}$ per $\mathrm{n} \mathrm{cm}^{-2} \mathrm{~s}^{-1}$, which is comparable to the neutron sensitivities of $\mathrm{BF}_{3}$ or ${ }^{3} \mathrm{He}$ detectors, i.e. around 4 and between $10-100 \mathrm{cps}$ per $\mathrm{n} \mathrm{cm}^{-2} \mathrm{~s}^{-1}$. 


\section{FUTURE WORK}

\section{A. Large sample irradiation facility}

In 2016, in the framework of the AIDA-II collaboration project, a new irradiation device was designed and installed in the Tangential channel, enabling irradiations of larger samples (up to $14 \mathrm{~cm}$ in diameter) at relatively intense neutron flux levels, of the order of $10^{12} \mathrm{n} \mathrm{cm}^{-2} \mathrm{~s}^{-1}$. The requirements for the irradiation device were to allow for easy insertion and withdrawal of samples, the capability of on-line irradiation testing of electronic components and temperature control (i.e. the provision of cable and coolant line feedthrough capability) and to ensure adequate shielding from neutron and gamma radiation originating from the reactor core. The design of the device was determined on the basis of experimental data and Monte Carlo particle transport calculations with the MCNP code. It consists of an aluminium inner channel liner and a trolley, onto which a cylindrical neutron shield made from highdensity borated polyethylene and a thick lead gamma shield are mounted.

To obtain information on the neutron field in the channel, measurements were performed of the ${ }^{197} \mathrm{Au}(\mathrm{n}, \gamma){ }^{198} \mathrm{Au}$ reaction rates, using $\mathrm{Al}-0.1 \% \mathrm{Au}$ material, and of the $1-\mathrm{MeV}$ NonIonizing Energy Loss (NIEL) equivalent neutron flux, using PIN diodes. The total neutron flux obtained from the activation measurements at full reactor power of $250 \mathrm{~kW}$ is $2.67 \times 10^{12}(1$ $\pm 0.027) \mathrm{n} \mathrm{cm}^{-2} \mathrm{~s}^{-1}$, the variation in the neutron flux over \pm 10 $\mathrm{cm}$ in the longitudinal direction is around $-11 \%$ and the variation over $\pm 5 \mathrm{~cm}$ in the vertical direction is $-4 \%$ at most. The NIEL equivalent flux scaled to full reactor power was found to be $(3.9 \pm 0.4) \times 10^{11} \mathrm{n} \mathrm{cm}^{-2} \mathrm{~s}^{-1}$.

In 2019 preparations were started for the installation of a similar irradiation facility in the adjacent irradiation channel which pierces the Thermal column. The objective for the new irradiation device is to meet the demands for experimental testing of electronic data acquisition systems, developed for the nuclear industry. This channel has lower flux, i.e. on the order of $10^{10} \mathrm{n} \mathrm{cm}^{-2} \mathrm{~s}^{-1}$, however the gradients are lower thus allowing more homogeneous irradiation over larger distance. For example the variation of neutron flux over $30 \mathrm{~cm}$ is less than $10 \%$.

\section{B. High energy gamma ray irradiation facility}

As most of today's gamma irradiations are performed by ${ }^{137} \mathrm{Cs}(0.662 \mathrm{MeV})$ or ${ }^{60} \mathrm{Co}(1.1732$ and $1.3325 \mathrm{MeV})$ sources, an irradiation facility with high energy gamma rays would be of high importance from the standpoint of material testing to assess degradation and deposited nuclear heat. This will become crucial in the future fusion nuclear devices, which consider water as a cooling fluid. The emitted radiation would cause increased doses to personnel [29] and also additional nuclear heating to various components such as superconducting coils cooled by liquid helium [30] [31]. The most important isotope in activated water is ${ }^{16} \mathrm{~N}$ produced via the ${ }^{16} \mathrm{O}(\mathrm{n}, \mathrm{p}){ }^{16} \mathrm{~N}$ reaction due to emission of gamma rays with energy of 6.13 $\mathrm{MeV}$ and $7.11 \mathrm{MeV}$ and half-life of $7.13 \mathrm{~s}$.

As there are only a few sources of high energy gamma rays an irradiation facility utilizing activated water as a source of high energy gamma rays is proposed at the JSI TRIGA Mark II research reactor. A conceptual design of such facility consists of a closed water loop leading the activated water from one of the reactor's irradiation channels into a shielded irradiation facility outside of the reactor core. As the proposed system is still in the design phase the activation of water inside a closed water loop system has to be calculated. In order to calculate the water activity, one must know the water flow rate, irradiation and cooling times, as well as water activation reaction rates inside the JSI TRIGA irradiation channels, which are calculated using the MCNP code.

Two different conceptual designs for the activated water irradiation facility are currently under consideration at the JSI TRIGA reactor. The first concept utilizes the Central channel for water activation while the second concept utilizes the Radial piercing port, which penetrates the concrete structure and the graphite reflector. Preliminary calculations to support final design show that the reaction rates for water activation are an order of magnitude higher in the Central channel, compared to the Radial piercing port, however the pipe system in the first case (length of $15 \mathrm{~m}$ ) would have to extend to the reactor water tank across the reactor platform and back down, which would result in a longer transit time of the activated water, compared to the radial port of pipe length of around $3 \mathrm{~m}$. Due to this the

${ }^{16} \mathrm{~N}$ decay rate in the Central channel case is only twice as high the one in the Radial port case.

The preliminary analysis shows that the conceptual design utilizing the Radial piercing port currently presents the best option for the irradiation facility due to the simpler design of the irradiation loop and comparable number of ${ }^{16} \mathrm{~N}$ decay rates in comparison to the design utilizing the central channel, which would require additional shielding to limit the dose rates in the reactor hall during operation. Analysis of irradiation facility design shows satisfactory intensity of high energy (6 and 7 $\mathrm{MeV}$ ) gamma ray source in the order of $10^{8} / \mathrm{s}$ decay rate thus producing dose rate of $2 \mathrm{mGy} / \mathrm{h}-4 \mathrm{mGy} / \mathrm{h}$.

\section{Pulse experiments}

A bilateral CEA-JSI project is currently underway, aimed at demonstrating the possibility of nuclear instrumentation testing at the JSI TRIGA reactor in reactor pulse mode. In steady state mode, the reactor can achieve a thermal power level of $250 \mathrm{~kW}$ and a maximum neutron flux level of around $2 \times 10^{13} \mathrm{n} \mathrm{cm}^{-2} \mathrm{~s}^{-1}$, however, pulse mode, the reactor is made prompt supercritical, resulting in a pulse in the reactor power with a duration ranging from around $5 \mathrm{~ms}$ to $1 \mathrm{~s}$. The highest peak power can reach 1 $\mathrm{GW}$, therefore the highest attainable flux is nearly $10^{17} \mathrm{n} \mathrm{cm}^{-2}$ $\mathrm{s}^{-1}$, albeit for a short amount of time. Measurements will be performed with several absolutely calibrated miniature ${ }^{235} \mathrm{U}$ fission chambers and a state-of-the art electronic acquisition system, named MONACO, developed by the CEA.

The expected project results will enhance the possibilities 
offered at the JSI TRIGA reactor for detector development and experimental testing. Furthermore, increased opportunities may arise for the reactor as a reference irradiation facility, further increasing its utilization.

\section{CONCLUSION}

The JSI TRIGA Mark II is a good example of a relatively small research reactor having a rather low neutron flux that can support a variety of high level research and development activities.

More than a decade of efforts in experimental and computational characterisation of the neutron and gamma fields in the irradiation facilities resulted in the JSI TRIGA reactor being a very well characterised neutron irradiation facility. For this reason it has become the reference centre for neutron radiation hardness studies for the CERN's LHC and other accelerators within the H2020 project AIDA II (Advanced European Infrastructures for Detectors at Accelerators). Several companies and research institutions have been using the reactor for testing of radiation hardness of their products, such as data acquisition and processing systems, cameras, LED lighting and similar. In addition, the reactor has been used for testing various detectors such as fission chambers and self-powered neutron detectors (SPNDs) as well as innovative data acquisition systems and methods developed and used by the CEA.

The JSI TRIGA reactor can significantly contribute to the development of new methods and knowledge in physics of nuclear detectors, radiation hardness studies as well as testing of nuclear instrumentation in a well characterised environment.

\section{REFERENCES}

[1] Peršič, A., et al., Nucl. Eng. Des. 318 (2017) 24-34.

[2] Goorley, T., MCNP6.1.1-Beta Release Notes. LA-UR-14-24680, 2014.

[3] Ravnik M. and Jeraj R., Research reactor benchmarks, Nucl. Sci. Eng., 145 (2003) 145-152.

[4] Štancar, Ž., et al., TRIGA-FUND-RESR-002, IRPHE2018-HANDBOOK, 2017.

[5] Snoj, L., et al., Appl. Radiat. Isot. 69 (2011) 136-14.

[6] Štancar, Ž., et al., Ann. Nucl. Energy, 112 (2018) 94-108.

[7] Grégoire, G., et al., First Analysis of the 2011 Joint CEA/IJS Reactor Dosimetry Campaign in Triga Mark-II Reactor: a Step for Preparing an Unfolding Dataset Available to Reactor Dosimetry Community, Proc. 21st Int. Conf. NENE, 2012.

[8] Radulović, V., et al., Use of boron nitride for neutron spectrum characterization and cross-section validation in the epithermal range through integral activation measurements, Nucl. Inst. Meth. A, 840 (2016) 5-14.

[9] Trkov, A., et al., The GRUPINT neutron spectrum adjustment code general features and characterization of the spectra in three irradiation channels of the JSI TRIGA reactor, 16th International Symposium on Reactor Dosimetry - ISRD16, 2017.

[10] Destouches, C., et al., The ${ }^{117} \mathrm{Sn}\left(\mathrm{n} . \mathrm{n}^{\prime}\right)^{117 \mathrm{~m}} \mathrm{Sn}$ reaction: a suitable candidate to investigate the epithermal neutron spectrum by reactor dosimetry techniques, Proc. 26th Int. Conf. NENE, 2017.

[11] Goričanec, T., et al., Ann. Nucl. Energy, 111 (2018) 407-440.

[12] Goričanec, T., et al., Appl. Radiat. Isot. 104 (2015) 34-42.

[13]Žerovnik, G., et al., Appl. Radiat. Isot. 96 (2015) 27-35.

[14] Štancar, Ž., et al., Nucl. Eng. Des. 325 (2017) 78-89.

[15]Radulović, V., et al., Appl. Radiat. Isot. 84 (2014) 57-65.

[16]Radulović, V., et al., Nucl. linstr.Meth. A, 804 (2015) 149-154.

[17] Fourmentel, D., et al., Delayed gamma measurements in different nuclear research reactors bringing out the importance of their contribution in gamma flux calculations. IEEE transactions on nuclear science, 63 (2016) 2875-2879.
[18] Gruel, A., et al., Gamma-heating and gamma flux measurements in the JSI TRIGA reactor, results and prospects, ANIMMA 2019.

[19]Ambrožič, K., et al., Nucl. Instrum. Meth. A, 911 (2018) 94-103.

[20] Ambrožič, K., et al., JSIR2S code system for delayed radiation field calculations, Proc. 27th Int. Conf. NENE, 2018.

[21]KRAMBERGER, G., et al., Nucl. Instrum. Meth. A, 476 (2002) 645-651.

[22] http://aida2020.web.cern.ch/

[23] KRAMBERGER, G., et al., Nucl. Instrum. Meth. A, 571 (2007) 608-611.

[24] Mandić, I. et al., Measurement of anomalously high charge collection efficiency in $\mathrm{n}+\mathrm{p}$ strip detectors irradiated up to $10 \mathrm{e} 16 \mathrm{neq} / \mathrm{cm} 2$, Nucl. Instrum. Meth. A, 603 (2009) 263-267.

[25]https://www.dito-lighting.com

[26] http://www.isec.se/

[27] Villard, J.F., et al., Improvements in neutron and gamma measurements for Material Testing Reactors, RRFM/IGORR 2016.

[28]Barbot, L., et al., MONACO v2: Multipurpose and Integrated Data Acquisition System for On-line Neutron and Gamma Measurements, ANIMMA 2019.

[29]Žohar, A., et al., On the Dose Fields due to Activated Cooling Water in Nuclear Facilities, Prog. Nucl. Energy, submitted for publication.

[30]Žohar, A., et al., Gamma Dose Field due to Activated Cooling Water in a Typical PWR, Proc. 26th Int. Conf. NENE, 2017.

[31] Iida, H., et al., 17th IEEE/NPSS Symposium Fusion Engineering (1997) $837-840$. 